\title{
Cerebral Venous Sinus Thrombosis After Cervical Spine Epidural Injection
}

\author{
Anthony F. Arguija $a^{a, b}$
}

\begin{abstract}
Headache is a common presenting complaint to the emergency department. Benign causes must be differentiated from life threatening causes. Cerebral venous thrombosis is a rare disease that has significant morbidity. This is a case report of a 36-year-old female who presented to multiple emergency departments within the span of 1 week after having a cervical spine epidural injection for her chronic neck pain. She was told repeatedly by multiple emergency departments that she had a spinal headache, but was ultimately diagnosed with cerebral venous sinus thrombosis. This case demonstrates a rare disease presenting with a common complaint and highlights the importance of considering alternative diagnoses and maintaining a broad differential diagnosis for headaches, especially in the face of an obvious cause.
\end{abstract}

Keywords: Cerebral venous sinus thrombosis; Post-dural puncture headache; Spinal headache; Headache

\section{Introduction}

Headache is a very common emergency department complaint. Differentiating benign causes from more serious causes can be difficult. Cerebral venous sinus thrombosis is a very rare disease that must be considered when evaluating a patient with headache.

\section{Case Report}

A 36-year-old African-American female with a history of migraine headaches and cervical radiculopathy presented to

\footnotetext{
Manuscript accepted for publication October 26, 2012

a'Long Beach Memorial Medical Center, Department of Emergency Medicine, Long Beach, California, USA

${ }^{\mathrm{b}}$ Corresponding address: Long Beach Memorial Medical Center, Department of Emergency Medicine, 2801 Atlantic Ave Long Beach, California, 90806, USA. E-mail: aarguija@gmail.com
}

doi: http://dx.doi.org/10.4021/jmc924w the emergency department (ED) for a third time in one week with a chief complaint of headache, photophobia and neck pain. She had been seeing a pain management specialist for her chronic neck pain sustained year's earlier secondary to a work related injury. She had undergone a cervical spine epidural injection one week prior. The headache began one day after the epidural. She reports numbing, tingling, photophobia and neck pain. She rated the pain as constant and severe, and nothing had been able to alleviate the pain. She reports going to a local ED when the headache first began. She was told that she had a spinal headache, given Fiorinal and Vicodin for her pain and told to follow-up with her pain management doctor. She was discharged home but states the pain continued and did not get better. Two days later, she went to another ED for persistent pain. At that ED she was given IV fluids and pain control and told that she would need a blood patch, and to follow-up with her pain management doctor. Two days later she presented to our ED for her third visit. She complained of a severe aching headache, mostly on the right. She also reported neck pain, tingling to her right upper extremity, photophobia and nausea. She denied fever, blurry vision, slurred speech, trauma, chest pain, shortness of breath, cough, or abdominal pain. She was scheduled to have a blood patch the following week, but stated the pain was too intense to wait.

She has a history of migraine headaches and cervical radiculopathy. No previous surgeries. She has no allergies to medications. She is only taking Vicodin and Fiorinal. No history of oral contraceptive pill (OCP) use. She denies tobacco, alcohol or drugs use. She has no known family history.

Her vitals: HR 40 - 48, Temp 98.2, BP 101/57, RR 18, $\mathrm{O}_{2} 100 \%$ on room air. She appears uncomfortable, requesting the lights be left off. Pupils are reactive bilaterally. Fundi disk margins are blurred. Conjunctiva is non-injected. Extraocular muscle are intact, no nystagmus. Neck is soft and supple but does have mild tenderness to palpation to the lower cervicals, no erythema or ecchymosis noted. Cardiovascular examination revealed sinus bradycardia with no murmurs. Lungs were clear bilaterally. Abdomen was soft and nontender. Extremities had full range of motion without gross deformities, no edema or cyanosis noted. No bruising or rashes noted to the skin. She was awake, alert and oriented. 


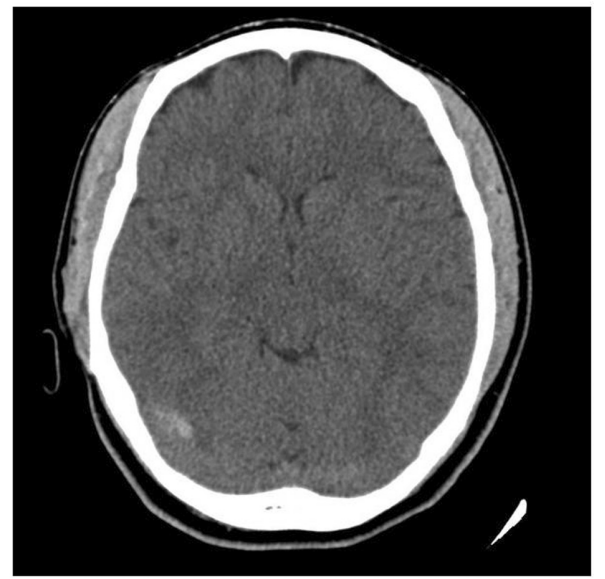

Figure 1. CT head without contrast: $2 \mathrm{~cm}$ area of increased attenuation on the right adjacent to the superior aspect of the right tentorium.

Fluent speech with normal comprehension and repetition. No facial asymmetry. Tongue is midline. Motor strength is $4 / 5$ on the right upper and lower extremity and $5 / 5$ on the left side. Babinski's were down-going bilaterally. Reflexes $2+$ throughout. Hyperesthesia noted to the right lower extremity.

CT head without contrast reveals a $2 \mathrm{~cm}$ area of increased attenuation on the right adjacent to the superior aspect of the right tentorium (Fig. 1).

Magnetic Resonance Imaging (MRI)/Magnetic Resonance Angiography (MRA)/Magnetic Resonance Venography (MRV) brain reveals dural venous sinus thrombosis in bilateral transverse sinuses, right worse than left, as well as in the straight sinus. No intracranial hemorrhage. No stenosis of the major intracranial vessels. No aneurysm or highflow vascular malformation.

MRI C-spine reveals no epidural hematoma. There is disc bulging at $\mathrm{C} 4 / 5$ through $\mathrm{C} 6 / 7$ with mild cord indententation but no gross abnormality. Osteophytosis causing mild to moderate left foraminal stenosis and mild right foraminal stenosis at $\mathrm{C} 5 / 6$ and mild bilateral foraminal stenosis at C4/5. Laboratory: $\mathrm{CBC}$, chemistry, UA are unremarkable. Pregnancy- negative. ECG, sinus bradycardia.

The patient was given IV fluids and narcotic pain medication with moderate improvement. She also remained bradycardic. She was admitted to the ICU. Consultation from neurology, neurosurgery, cardiology and hematology were obtained. A hypercoagulable work-up was performed which was negative for protein $\mathrm{C}$ and $\mathrm{S}$ deficiency, Factor $\mathrm{V}$ Leiden, cardiolipin, and lupus anticoagulant; but did reveal a factor VIII level $>300 \%(<100 \%)$. She was started on intravenous heparin and transitioned to coumadin. Her hospital stay was complicated by right lower lobe pulmonary emboli as well as a right upper extremity DVT. Her bradycardia was felt to be secondary to increased intracranial pressure and was started on Diamox. Her heart rate gradually improved to $70-80 \mathrm{~s}$ during her hospital stay. Her headache persisted, with only mild-moderate improvements. Her neurological examination remained unchanged. Pain management was consulted as well as physical medicine and rehabilitation. She was eventually transferred to a rehabilitation facility.

\section{Discussion}

Cerebral venous sinus thrombosis (CVT) is very uncommon, with an estimated annual incidence of 3-4 cases per million [1]. It occurs more commonly in younger women than in men and is much more common during pregnancy [2]. Pregnancy and the puerperium, oral contraceptive use, prothrombotic conditions, malignancy and infection are all major risk factors associated with CVT [1,2]. Headache is the most common presenting symptom. It may be difficult to distinguish it from other common causes of headache such as migraine headache or post dural puncture headache. Visual disturbances, motor weakness, and seizures are other common symptoms associated with CVT [2]. The mechanism responsible for CVT is complex, but involves increased venous pressure secondary to dural sinus obstruction which leads to decreased cerebral blood flow and cerebral edema which results in leakage of blood into the parenchymal space [1].

Although rare, CVT has been associated with dural puncture. In the International study on Cerebral venous and dural sinus thrombosis (ISCVT) lumbar puncture was associated with CVT in only $1.9 \%$ [2]. In a much smaller retrospective study of 66 patients, dural puncture was listed as the fourth most frequent risk factor associated with CVT [3]. A few case reports of CVT during pregnancy following failed epidural and blood patch have also been described [4]. Pregnancy is a well known hypercoagulable state and risk factor for CVT. In several case reports, the patient had more than one risk factor. In the ISCVT cohort, $43 \%$ had greater than one risk factor. In this case, our patient was not pregnant and not on OCP's as a majority of cases tend to be. She was female and had a mechanical precipitant (cervical spine epidural with likely dural puncture) which is a known risk factor for CVT. To my knowledge, no case reports have been published documenting CVT post cervical spine epidural injection.

Complicating the clinical picture was a previously unknown hypercoagulable state. Elevated factor VIII levels have been identified as an independent risk factor for venous thromboembolism, and are recognized as a strong thrombotic risk factor in the African-American population [5-8]. There is also evidence to suggest that individuals with elevated factor VIII levels are at increased risk for recurrent venous thromboembolism [6]. This underlying hypercoagulable state in conjunction with another known risk factor, dural puncture, likely led to the development of CVT in this 
patient. The postulated mechanism by which dural puncture may contribute to CVT has been theorized by Canhao et al as follows: Dural puncture causes a decrease in CSF volume and pressure which causes a compensatory venodilation which leads to increased blood volume and decreased blood flow which ultimately may lead to venous thrombosis [9].

Despite the complicating clinical scenario, a diagnosis was reached and this patient received appropriate anticoagulation and pain control. She did have a prolonged hospital course complicated by pulmonary emboli and DVT of the upper extremity despite being on anticoagulation. Her headaches persisted but she did not have any decline in her neurological function. She was eventually discharged to a rehabilitation facility. Her prognosis remains uncertain; however, according to the ISCVT cohort, approximately $80 \%$ of individuals with CVT had a complete recovery at 16 month follow-up and $13.6 \%$ resulted in death or dependence [2].

\section{Conclusion}

Cerebral Venous Sinus Thrombosis is a rare cause of headaches, but one that has significant morbidity. This case highlights the importance of broadening the differential diagnosis, considering alternative explanations, and not narrowing the focus of a seemingly obvious cause for a patient's symptoms.

\section{Competing Interests}

No conflicts of interests or sources of funding.

\section{References}

1. Stam J. Thrombosis of the cerebral veins and sinuses. N
Engl J Med. 2005;352(17):1791-1798.

2. Ferro JM, Canhao P, Stam J, Bousser MG, Barinagarrementeria F. Prognosis of cerebral vein and dural sinus thrombosis: results of the International Study on Cerebral Vein and Dural Sinus Thrombosis (ISCVT). Stroke. 2004;35(3):664-670.

3. Wilder-Smith E, Kothbauer-Margreiter I, Lammle B, Sturzenegger M, Ozdoba C, Hauser SP. Dural puncture and activated protein $\mathrm{C}$ resistance: risk factors for cerebral venous sinus thrombosis. J Neurol Neurosurg Psychiatry. 1997;63(3):351-356.

4. Barrett J, Alves E. Postpartum cerebral venous sinus thrombosis after dural puncture and epidural blood patch. J Emerg Med. 2005;28(3):341-342.

5. O’Donnell J, Tuddenham EG, Manning R, KemballCook G, Johnson D, Laffan M. High prevalence of elevated factor VIII levels in patients referred for thrombophilia screening: role of increased synthesis and relationship to the acute phase reaction. Thromb Haemost. 1997;77(5):825-828.

6. Kraaijenhagen RA, in't Anker PS, Koopman MM, Reitsma PH, Prins MH, van den Ende A, Buller HR. High plasma concentration of factor VIIIc is a major risk factor for venous thromboembolism. Thromb Haemost. 2000;83(1):5-9.

7. Tsai AW, Cushman M, Rosamond WD, Heckbert SR, Tracy RP, Aleksic N, Folsom AR. Coagulation factors, inflammation markers, and venous thromboembolism: the longitudinal investigation of thromboembolism etiology (LITE). Am J Med. 2002;113(8):636-642.

8. Patel RK, Ford E, Thumpston J, Arya R. Risk factors for venous thrombosis in the black population. Thromb Haemost. 2003;90(5):835-838.

9. Canhao P, Batista P, Falcao F. Lumbar puncture and dural sinus thrombosis--a causal or casual association? Cerebrovasc Dis. 2005;19(1):53-56. 